Ann. Génét. Sél. anim., I970, 2 (4), 449-456.

\title{
EXPRESSION DE LA VARIABILITÉ GÉNÉTIQUE ENTRE ÉTABLES DE SÉLECTION ET ENTRE LIGNÉES PATERNELLES DANS L'APTITUDE GÉNÉRALE AU CROISEMENT DES MÂLES DE RACE A VIANDE
}

\author{
J.-C. MOCQUOT \\ Station de Génétique quantitative et appliquée, \\ Centre national de Recherches zootechniques, 78-Jouy-en-Josas \\ Institut national de la Recherche agronomique
}

RÉSUMÉ

Les méthodes classiques d'estimation de la variance génétique entre étables sont basées soit sur le calcul de la variance des productions moyennes des lots de demi-sœurs et de contemporaines non apparentées d'une même étable, soit sur la relation entre les productions moyennes de ces deux groupes d'individus. Elles impliquent de ce fait l'existence de contemporains contrôlés dans les programmes de testage. En outre la signification des résultats obtenus reste fragmentaire lorsque la population de mâles mis au testage est indépendante de celle des femelles utilisées pour ce testage. C'est le cas par exemple du contrôle de descendance des mâles de race à viande produits dans les élevages de sélection lorsque ce contrôle est réalisé dans des élevages commerciaux sur des descendants croisés. D'une façon plus générale, certains centres d'insémination situés dans des zones d'extension des races assurent souvent l'essentiel de leur approvisionnement en géniteurs mâles par des achats d'animaux produits hors de leur zone dans des élevages de sélection.

Pour pallier ces limites des méthodes classiques, une méthode directe basée sur l'analyse de la variance des index génotypiques entre élevages d'origine des taureaux et intra élevages est proposée. Lorsque ces élevages pratiquent la monte naturelle on peut également considérer les lignées paternelles intra élevage d'origine comme deuxième source de variation dans un schéma hiérarchique à deux voies. Les équations d'estimation de la variance génétique entre étables d'origine et entre lignées paternelles sont établies à partir des résultats de cette analyse de variance.

Une application de cette méthode aux index des taureaux limousins sur le poiđs à 75 jours de leurs descendants croisés permet de constater que l'étable d'origine intervient pour 20 p. 100 alors que l'origine paternelle n'intervient que pour 10 p. 100 dans la variance génétique de ce critère. Le premier chiffre est conforme à ce que l'on obtient en production laitière compte tenu de l'héritabilité et de la part génétique des différences entre troupeaux.

\section{INTRODUCTION}

Le choix rationnel des reproducteurs mâles à soumettre au contrôle de descendance implique de connaître l'importance relative des différences entre les niveaux génétiques moyens des étables et des lignées paternelles dont ils sont issus. 
Il convient donc de disposer de méthodes permettant de déterminer la part relative de chacun de ces deux facteurs dans la variance génétique des performances de ces reproducteurs.

Deux types de méthodes, citées par PIRChNer et Lush (I959) sont couramment utilisées pour estimer la part génétique de variance de la production laitière liée à l'étable. Dans la première méthode (ROBERTSON et RENDEL, I954) cette estimation est dérivée du coefficient de régression $(m)$ de la production des filles de taureaux utilisés en insémination artificielle sur la production des génisses contemporaines entretenues dans les mêmes étables. En effet $m$ est égal à I s'il n'existe pas de différences génétiques entre étables, à 0,5 si ces différences sont d'origine purement génétique. Dès lors l'estimation de la part génétique des différences entre étables est égale à $2(\mathrm{I}-m)$. Dans la deuxième méthode (Mc GilliarD, I952) on compare les composantes "étables " de la variance du caractère, pour les demi-sœurs de père d'une part, pour les animaux contemporains non apparentés d'autre part. Dans le premier cas cette composante ne comporte que le quart de la variance génétique des différences entre étables et la totalité de la variance due au milieu, tandis que dans le second cas elle comporte en totalité la variance génétique et la variance milieu. Ces deux méthodes permettent d'estimer la part de la variance entre étables imputable à des différences de niveau génétique moyen entre ces étables. Si 1'on suppose estimées, par ailleurs, l'héritabilité du caractère et l'importance relative des différences entre étables par rapport à la variance phénotypique totale, on peut en déduire la part de la variance génétique totale due à des différences de niveau génétique moyen entre les étables.

Les études réalisées à partir de ces deux méthodes ou de méthodes dérivées font apparaitre, pour les caractères de production des bovins laitiers, une part génétique de variance entre étables de l'ordre de ro à $20 \%$ (ROBERTSON et RENDEL, i954; Pirchner et Lush, I959; Bereskin et Hazel, I962; VAN Vleck, I963; Poutous, r964). Ces résultats ont été confirmés par Boyazogl, , Poly et PouTous (I964) dans le cas de la production laitière des brebis soumises à la traite. Compte tenu d'un coefficient d'héritabilité de 0,3 et d'une importance relative des différences entre étables égale à $30 \%$ de la variance phénotypique totale, ces auteurs trouvent que Io à $20 \%$ de la variance génétique totale est imputable à des différences de niveau génétique moyen entre les étables.

Ces méthodes ne s'appliquent pas au cas des taureaux de race à viande utilisés principalement pour le croisement industriel du fait de la séparation assez nette existant entre les étables qui produisent et celles qui utilisent ces reproducteurs. Les premières sont situées dans le berceau de la race, utilisent la monte naturelle et produisent des bœufs ou des taurillons de race pure, les deuxièmes sont situées dans les zones d'extension de la race, utilisent l'insémination artificielle et produisent des veaux de boucherie par croisement industriel. On doit noter par ailleurs que pour ce dernier type d'étables, on ne contrôle qu'un nombre très limité de descendants, souvent un seul par étable, de sorte qu'il n'existe pratiquement pas d'animaux contrôlés contemporains.

La présente étude a pour but de présenter une méthode générale permettant d'estimer les différences génétiques relatives entre troupeaux d'origine des mâles 
et entre lignées paternelles pour l'aptitude générale des mâles au croisement industriel. Les hypothèses admises sont les suivantes :

Io Les valeurs génotypiques ainsi que les paramètres de la variabilité génétique estimés à partir des résultats du contrôle de descendance dans les troupeaux d'utilisation sont connus.

$2^{\circ}$ Le contrôle de descendance ne concerne qu'un seul produit par élevage de croisement et sensiblement le même nombre de produits par reproducteur soumis à ce contrôle.

$3^{\circ}$ La pratique de la monte naturelle dans les élevages de sélection permet de considérer les familles de demi-frères à l'intérieur de chaque élevage.

\section{MÉTHODE D'ÉTUDE}

La méthode proposée consiste à réaliser dans une première phase une analyse de variance des index génotypiques des géniteurs mâles (VIssAC B., I964) classés selon deux modalités : l'étable d'origine d'une part, la lignée paternelle intraétable d'autre part. Cette analyse permet de tester les effets de ces deux facteurs sur la variance des index. La deuxième étape de la méthode consiste à décomposer la valeur génotypique estimée de chaque individu et la variance génétique en leurs différentes composantes, estimables à partir du schéma expérimental, à savoir :

Io L'effet du niveau génétique moyen de l'étable d'origine.

$2^{\circ}$ L'effet du niveau génétique de la lignée paternelle.

$3^{\circ}$ L'effet génétique résiduel.

$4^{\circ}$ L'effet aléatoire résiduel.

On peut ainsi estimer la part respective de chacun des trois premiers effets dans la variance génétique totale.

Nous appellerons :

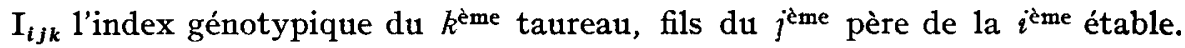

$i$ variant de I à $\alpha$.

$j$ variant de $\mathrm{r}$ à $\beta_{i}$.

$k$ variant de I à $\gamma_{i j}$.

$\mathrm{N}_{i}=\sum_{j=1}^{\beta i} \gamma_{i j}=1 \mathrm{e}$ nombre de taureaux dans la $i$ ème étable.

$\mathrm{N}_{. .}=\sum_{i=\mathrm{I}}^{\alpha} \mathrm{N}_{i} .=$ le nombre total de taureaux.

$\mathrm{I}_{i j .}=\sum_{k=\mathrm{I}}^{\gamma i j} \mathrm{I}_{i j k}$

$\mathrm{I}_{i . .}=\sum_{j=\mathrm{x}}^{\beta i} \mathrm{I}_{i j}$.

$\mathrm{I} \ldots=\sum_{i=1}^{\alpha} \mathrm{I}_{i .}$ 
On réalise une analyse de variance des index génotypiques selon le modèle suivant :

$$
\mathrm{I}_{i j k}=\mu+f_{i}+p_{i j}+t_{i j k}
$$

avec $f_{i}$ : effet étable d'origine,

$p_{i j}$ : effet père intra étable,

$t_{i j k}$ : effet taureau intra père intra étable,

analyse qui conduit au tableau $\mathbf{I}$.

TABLEAU I

Analyse de la variance des index génotypiques

\begin{tabular}{|c|c|c|}
\hline Origine de la variance & Somme des carrés centrés & Degrés de liberté \\
\hline 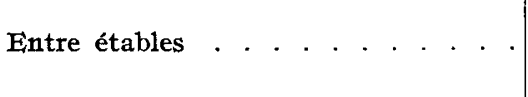 & $\mathrm{F}=\sum_{i} \frac{\mathrm{I}_{1 . .}^{2}}{\mathrm{~N}_{i .}}-\frac{\mathrm{I}_{\ldots . .}^{2}}{\mathrm{~N}_{. .}}$ & $\alpha-I$ \\
\hline Entre pères intra étables . . . . & $\mathrm{P}=\sum_{i j} \frac{\mathrm{I}_{i j .}^{2}}{\gamma_{i j}}-\sum_{i} \frac{\mathrm{I}_{i . .}^{2}}{\mathrm{~N}_{i .}}$ & $\sum_{i}\left(B_{i}-1\right)$ \\
\hline Entre taureaux intra pères intra étables & $\mathrm{I}=\sum_{i j k} \mathrm{I}_{i j k}^{2}-\sum_{i j} \frac{\mathrm{I}_{i j .}^{2}}{\gamma_{i j}}$ & $\sum_{i j}\left(\gamma_{i j}-\mathrm{I}\right)$ \\
\hline Total . . . . . . & $\mathrm{T}=\sum_{i j k} \mathrm{I}_{\imath j k}^{2}-\frac{\mathrm{I}_{\ldots .}{ }^{2}}{\mathrm{~N}_{. .}}$ & N.. $-\mathbf{I}$ \\
\hline
\end{tabular}

Il est alors possible de tester les deux effets étudiés.

- L'effet de l'étable d'origine $=\mathrm{F}\left[\alpha-\mathrm{I}, \sum_{i}\left(\beta_{i}-\mathrm{I}\right)\right]=\frac{\mathrm{F} /(\alpha-\mathrm{I})}{\mathrm{P} / \sum_{i}\left(\beta_{i}-\mathrm{I}\right)}$

- 1'effet du père intra étable $=\mathrm{F}\left[\sum_{i}\left(\beta_{i}-\mathrm{I}\right), \sum_{i j}\left(\gamma_{i j}-\mathrm{I}\right)\right]=\frac{\mathrm{P} / \sum_{i}\left(\beta_{i}-\mathrm{I}\right)}{\mathrm{I} / \sum_{i j}\left(\gamma_{i j}-\mathrm{I}\right)}$

Supposons maintenant que $I_{i j k}$ soit une variable normale de moyenne $\mu$ de variance $\sigma^{2}$ et dont les variations obéissent au modèle additif suivant :

$$
\mathrm{I}_{i j k}=\mu+2 b\left(f_{i}+p_{i j}+g_{i j k}+\frac{\sum_{l=1}^{n} r_{i j k l}}{n}\right)
$$


avec :

$f_{i}=$ influence génotypique due à la $i$ ème étable.

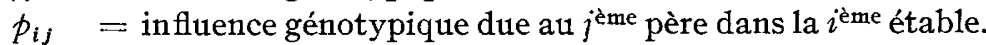

$g_{i j k}=$ influence génotypique restante.

$r_{i j k l}=$ influence résiduelle imputable à chacun des descendants intervenant dans l'estimation de la valeur génotypique.

$n=$ nombre de descendants ou coefficient de pondération dans le cas d'une "comparaison aux contemporaines " (supposé identique en première approximation pour tous les reproducteurs testés).

$b=$ coefficient de détermination de l'index caractérisant sa précision.

$b=\frac{n}{n+\left(4 / h^{2}-\mathrm{I}\right)}$.

On suppose en outre :

$\mathrm{E}\left(f_{i}\right)=\mathrm{E}\left(p_{i j}\right)=\mathrm{E}\left(g_{i j k}\right)=\mathrm{E}\left(r_{i j k l}\right)=0$.

$f^{2}=$ variance des $f_{i}$.

$p^{2}=$ variance des $p_{i j}$ ( $i$ fixé ou non).

$g^{2}=$ variance des $g_{i j k}$ ( $i$ et $j$ fixés ou non).

$r^{2}=$ variance des $r_{i j k l}$ ( $i, j, k$ fixés ou non).

$f_{i}, p_{i j}, g_{i j k}$ et $r_{i j k l}$ variables indépendantes.

Compte tenu de ces hypothèses, les espérances des sommes de carrés centrés s'écrivent :

$$
\begin{gathered}
\mathrm{E}(\mathrm{F})=4 b^{2}\left[\left(\mathrm{~N} . .-\frac{\sum_{i} \mathrm{~N}_{i .}^{2}}{\mathrm{~N}_{. .}}\right) f^{2}+\left(\sum_{i} \frac{\sum_{j} \gamma_{i j}^{2}}{\mathrm{~N}_{i .}}-\frac{\sum_{i j} \gamma_{i j}^{2}}{\mathrm{~N} . .}\right) p^{2}+(\alpha-\mathrm{I})\left(g^{2}+\frac{r^{2}}{n}\right)\right] \\
\mathrm{E}(\mathrm{P})=4 b^{2}\left[\left(\mathrm{~N}_{. .}-\sum_{i} \frac{\sum_{j} \gamma_{i j}^{2}}{\mathrm{~N}_{i .}}\right) p^{2}+\sum_{i}\left(\beta_{i}-\mathrm{I}\right)\left(g^{2}+\frac{r^{2}}{n}\right)\right] \\
\mathrm{E}(\mathrm{I})=4 b^{2}\left[\sum_{i j}\left(\gamma_{i j}-\mathrm{I}\right)\left(g^{2}+\frac{r^{2}}{n}\right)\right]
\end{gathered}
$$

En égalant ces expressions aux valeurs de F, P et I calculées à partir de l'échantillon on obtient un système de 3 équations à 4 inconnues :

$$
f^{2}, p^{2}, g^{2} \text { et } r^{2} \text {. }
$$

Si nous supposons en outre que l'héritabilité du caractère, estimée et connue par ailleurs, est égale à

$$
h^{2}=\frac{f^{2}+p^{2}+g^{2}}{f^{2}+p^{2}+g^{2}+r^{2}}
$$

on obtient une quatrième équation qui permet de résoudre le système et de calculer chacune de ces composantes. 
Les estimations, exprimées en pourcentage de la variance génétique totale, des différences de niveau génétique moyen entre les étables d'origine d'une part, des différences entre lignées paternelles d'autre part, sont respectivement :

$$
\mathrm{A}=\frac{f^{2}}{f^{2}+p^{2}+g^{2}} \times \text { I00 } \quad \text { et } \quad \mathrm{B}=\frac{p^{2}}{f^{2}+p^{2}+g^{2}} \times \text { IоO }
$$

\section{APPLICATION}

Une application de ce modèle a été faite dans le cas du schéma de sélection des mâles de race à viande sur la valeur de leurs veaux de boucherie croisés que nous avons rappelé ci-dessus.

\section{r. - Matériel animal.}

Le travail a porté sur les index des poids à âge de référence des taureaux de race limousine.

Les index avaient été calculés selon la méthode proposée par Vissac (1964) dans laquelle les taureaux sont testés, par séries contemporaines, sur le poids de leurs descendants à 75 ou 90 jours selon les séries. Cette fluctuation est liée aux variations régionales dans l'âge d'abattage des veaux de boucherie. Nous n'avons retenu que les taureaux testés sur plus de 30 descendants et appartenant à des séries de 5 individus au moins.

Dans le but d'éliminer l'influence des âges de référence différents suivant les séries, ainsi que les effets spécifiques de chaque série, les index de chacun des taureaux ont été exprimés en pourcentage de la moyenne des autres taureaux de leur série. Ceci peut présenter quelques inconvénients sur le plan statistique, mais permet par ailleurs de tenir compte d'un plus grand nombre de résultats.

On a ensuite recherché les étables d'origine de ces taureaux et conservé celles qui avaient fourni au moins 2 taureaux testés dans les ronditions énoncées ci-dessus. Les taureaux ont été regroupés à l'intérieur de chacune de ces étables selon leur origine paternelle. Il faut noter à ce propos que, pour notre échantillon de calcul, 2 taureaux fils d'un même père ne se trouvaient jamais dans 2 étables différentes (saillie naturelle dominante).

L'échantillon de calcul, ainsi constitué, comprenait 137 taureaux, issus de 38 étables et de 108 pères différents. Le nombre moyen de descendants contrôlés par taureau était de $n=60,4$.

\section{2. - Résultats.}

Un test de normalité effectué sur ces index exprimés en pourcentages permet d'assimiler leur distribution à une loi normale.

Les variances et leurs degrés de liberté s'établissent de la façon suivante :

- variance totale : $22,356(136)$;

- variance entre étables : 33,299 (37);

- variance entre pères, intra étables : 19,025 (70);

- variance entre taureaux, intra pères, intra étables : r6,389 (29).

Soit des valeurs de la statistique $\mathrm{F}$ :

- pour l'effet étables : $\mathrm{F}(37,70)=\mathrm{I}, 75$;

- pour l'effet pères : $\mathrm{F}(70,29)=\mathrm{I}, \mathrm{I} 6$.

Il existe donc des différences significatives entre les étables d'origine et entre les lignées paternelles en ce qui concerne la distribution des index des poids aux âges de référence. 
Si l'on suppose que $h^{2}=0,16$ (PoujARdieu et VisSac, I968) on est conduit au système suivant :

$$
\begin{aligned}
& 0=0,84 f^{2}+0,84, p^{2}+0,84 g^{2}-0, I 6 r^{2} \\
& 60 \mathrm{I}, 59 \mathrm{I}=\mathrm{I} 29.8 \mathrm{Io} f^{2}+49,746 p^{2}+37\left(g^{2}+\frac{r^{2}}{60,4}\right) \\
& 650,282=\quad 85,714 p^{2}+70\left(g^{2}+\frac{r^{2}}{60,4}\right) \\
& 232,069=\quad 29\left(g^{2}+\frac{r^{2}}{60,4}\right)
\end{aligned}
$$

et aux estimations des différentes composantes de la variance :

$$
\begin{aligned}
& f^{2}=\mathrm{II}, 95^{\circ} \\
& p^{2}=\mathrm{I}, 05^{2} \\
& g^{2}=7, \mathrm{I} 23 \\
& r^{2}=53, \mathrm{I} 56
\end{aligned}
$$

dès lors

$$
\mathrm{A}=\mathrm{I} 9,25 \% \text { (étables d'origine) }
$$

et

$$
\mathrm{B}=\text { I0,39 \% (lignées paternelles). }
$$

On constate donc que l'étable d'origine intervient pour $20 \mathrm{p}$. Ioo alors que l'origine paternelle n'intervient que pour Io $\mathrm{p}$. Ioo dans la variance génétique totale des poids à âges de référence des taureaux limousins. Le premier chiffre est tout à fait comparable aux estimations indirectes de la part de variance génétique de production laitière imputable à l'élevage d'origine que l'on pourrait faire d'après les valeurs d'héritabilité et de variances génétiques entre étables calculées par les auteurs (cf. introduction).

Une analyse détaillée des résultats obtenus par cette méthode sera fournie ultérieurement. Cependant, il faut dès maintenant noter que la valeur des estimations ainsi obtenues est conditionnée par le respect des hypothèses du modèle ce qui n'est pas toujours réalisé en pratique.

La précision de ces estimations dépend en outre du nombre de taureaux par étable, du nombre de lignées paternelles par étable, et du nombre de taureaux par lignée paternelle. Ces nombres sont en général réduits dans la pratique. Il faut noter à ce propos que la méthode ne permet pas de discriminer la part des différences génétiques entre étables imputable à une sélection délibérée de la part des éleveurs, de celle résultant de l'utilisation d'un très petit nombre de reproducteurs mâles d'une génération à l'autre. Ce dernier facteur tend à accroître les différences génétiques entre étables d'une fraction non utilisable puisque de sens et de valeur non connus.

Néanmoins les résultats obtenus semblent préciser l'intérêt qu'il faut accorder au niveau d'étable et à l'origine paternelle des taureaux de race à viande destinés au croisement industriel. 


\title{
CONCLUSION
}

Cette méthode d'estimation de l'importance génétique de l'origine des reproducteurs mâles à soumettre au contrôle de descendance possède en définitive un caractère de généralité que n'avaient pas les procédés indirects utilisés jusqu'ici et que nous avons rappelés sommairement.

En particulier, elle permet de considérer les situations où l'ensemble du cheptel des élevages dont on analyse la variabilité génétique n'est pas soumis au contrôle et d'explorer la variabilité entre les troupeaux de sélection ainsi qu'entre leurs lignées mâles à partir des données obtenues en zone d'extension.

Reçu pour publication en octobre 1970.

\section{SUMMARY}

\author{
EXPRESSION OF GENETIC VARIABILITY BETWEEN SELECTION STABIES AND \\ SIRE IINES IN THE GENERAL CROSSING ABILITY OF BEEF-BREED MALES.
}

Classical methods of estimating genetic variance between stables are based either on computation of the variation in average production of lots of half sisters and unrelated contemporaries of the same stable, or on the relationship between the average production of these two groups of individuals. This means that contemporaries are examined in testing programs. Moreover, the significance of results obtained is fragmentary when the male population tested in independent of that of the female used for this testing. This is true of progeny-tested beef-breed males produced on selection farms when testing is done on crossed progeny by commercial breeders. In a more general way, some artificial insemination centers located in breed extension areas often insure the main part of their stock of male breeders by buying animals produced on selection farms outside their area.

In order to improve these classical methods, we propose a direct method based on an analysis of the variation between farm-of-origin and intra-farm genotypic index. When these farms use natural mating, paternal descendants of intra-farm origin may alos be used as a second source of variation in a two-way hierarchic plan. Equations of estimation of genetic variance between stable of origin and paternal descendants are established from results of this variance analysis.

When this method is applied to the Limousin bull index on the weight of their crossed descendants at 75 days, it is evident that the stable of origin is responsible for $20 \%$, while paternal origin accounts for only $10 \%$ of the genetic variance of this criterion. The former figure agrees with that obtained for milk production, taking into account heritability and the genetic proportion of differences between herds.

\section{RÉFÉRENCES BIBLIOGRAPHIQUES}

Berfskin B., Hazel I. N., I962. Role of herd averages in dairy sire evaluation. J. Anim. Sci., 21, 969. Boyazoglu J. G., Poly J., Pourous M., I964. Aspects quantitatifs de la production laitière des brebis. II. Estimation des différences d'origine génétique et non génétique entre troupeaux. Annls. Zootech, 13, 289-297.

MC GILLIARD I. D., 1952. Usefulness of the herd average in estimating breeding values of dairy cattle. Ph. D. Thesis, Iowa State College Library, Ames, Iowa (U.S.A.) (cité par PIRchNer et LuSH, 1959).

Prrchner F., LUSH J. I.., I959. Genetic and environmental portions of variation among herds in butterfat production. J. Dairy Sci., 42, II5-122.

Poujardieu B., Vissac B., 1968. Étude biométrique de la valeur bouchère de veaux croisés charolais, et limousin. I. Paramètres génétiques et phénotypiques. Annls. Zootech., 17, I 43-I 57.

Poutous M., 1964. (Données non publiées).

Robertson A., RENDEL J. M., 1954. The performance of heifers got by artificial insemination. J. Agric. Sci., 44, I84-192.

VAN VLECK I. D., I963. Regression of records on herd mate averages. J. Dairy. Sci., 46, 846-849.

VISSAC B., I964. Méthode de détemination de l'index génotypique des taureaux d'insémination sur la valeur de leurs veaux de boucherie. Annls, Zootech., 13, 267-275. 